1. Instituto de Atención Pediátrica,

2. Universidad Autónoma de Ciencias Médicas.

Abreviaturas: $A B C$, área bajo la curva; $\mathrm{CMI}$, concentración mínima inhibitoria; $C$ max, Concentración máxima; CPM, concentración preventiva de mutantes; EPA, efecto postantibiótico; MEF, fluido del oído medio; PISP, S. pneumoniae con susceptibilidad intermedia a penicilina; PRSP S, pneumoniae resistente a penicilina; PSSP $S$, pneumoniae susceptible a penicilina; OMA, Otitis media aguda; Pd, farmacodinamia; Pk, farmacocinética; VSM, ventana de selección de mutantes.

Correspondencia: $\mathrm{Dr}$ Adriano Arguedas Mohs. Instituto de Atención Pediátrica.

Apartado Postal 607-II50 La Uruca, San José, Costa Rica. Correo electrónico: aarguedas@iped.net

ISSN 0001-6002/2006/48/3/113-118 Acta Médica Costarricense, @2006 Colegio de Médicos y Cirujanos

\section{Principios farmacocinéticos y farmacodinámicos en el tratamiento de niños con otitis media}

\author{
Lara Aguilar-Morales ', Carolina Soley-Gutiérrez ', Adriano Arguedas-Mohs 1,2.
} Cor

\begin{abstract}
Resumen
Debido a que la otitis media aguda, es la primera causa de uso de antibióticos en la edad pediátrica, es importante lograr una utilización adecuada y racional de los antibióticos en estas patologías, siendo imprescindible que antes de iniciar un tratamiento antimicrobiano se haga un buen diagnóstico clínico y se conozca el patrón microbiológico y de susceptibilidad antimicrobiana prevaleciente. La presente revisión incorpora conceptos novedosos para seleccionar la terapia antimicrobiana en niños con otitis media, tomando en cuenta principios farmacocinéticos y farmacodinámicos aplicados a conceptos microbiológicos. Estos nuevos conceptos han revolucionado el tratamiento de diversos procesos infecciosos en pediatría y superan los criterios, un poco más simples, en los que se define únicamente si una bacteria es sensible o resistente a un determinado antibiótico, incorporando aspectos fundamentarles como lo son la biodisponibilidad, la penetración del antimicrobiano al oído medio, la dosis recomendada y los intervalos entre cada dosificación.
\end{abstract}

Descriptores: farmacocinética, farmacodinamia, otitis media, erradicación.

Key words: pharmacokinetic, pharmacodynamic, otitis media

Recibido: 8 de noviembre de 2005 Aceptado: 28 de marzo de 2006

Las infecciones respiratorias agudas constituyen la primera causa de consulta médica en la edad pediátrica y, dentro de ellas, la otitis media aguda (OMA) es la patología más común y la causa más frecuente de uso de antibióticos en la infancia. La enfermedad tiene su máxima incidencia entre los 6 y 9 meses de edad y se reporta que al año de edad, aproximadamente el $75 \%$ de los niños ha sufrido al menos un episodio de OMA y el $15 \%$ ha presentado $\geq 3$ episodios ${ }^{1-4}$.

Los gérmenes que con mayor frecuencia se aíslan del oído medio en niños con otitis media son el Streptococcus pneumoniae, el Haemophilus influenzae, la Moraxella catarrhalis y el Streptococcus pyogenes. El patrón de resistencia antibiótica de estas bacterias varía según de la región geográfica, y es la base fundamental para establecer recomendaciones terapéuticas ${ }^{5}$. La acción de un antimicrobiano depende de múltiples factores, siendo los más importantes la acción antibacteriana específica (efecto bacteriostático o bactericida) y la sensibilidad del microorganismo a este antibiótico. También es importante conocer el proceso desde que se administra el antimicrobiano hasta que llega al tejido infectado (farmacocinética) y la acción del antibiótico en el sitio de infección (farmacodinamia) ${ }^{6}$. Los descubrimientos en este campo han identificado puntos de corte que predicen la erradicación bacteriana, el éxito clínico e incluso la prevención de selección de resistencia ${ }^{7}$. 
El motivo de esta revisión es analizar la importancia clínica de los conceptos de farmacocinética y farmacodinamia en el tratamiento de la otitis media.

\section{Otitis media}

Actualmente la microbiología del oído medio de niños costarricenses con otitis media demuestra que los gérmenes que con mayor frecuencia producen infecciones en Costa Rica son el S. pneumoniae (49\%), el H. influenzae (37\%), el $S$. pyogenes (7\%) y la $M$. catarrhalis (7\%). El $7 \%$ y el 93\% de los $H$. influenzae y de las $M$. catarrhalis, respectivamente, son cepas productoras de $\beta$-lactamasas ${ }^{8}$.

En términos generales, los episodios de OMA se curan espontáneamente; sin embargo, en el caso de OMA producida por $S$. pneumoniae el porcentaje de resolución espontánea es de tan solo un 16\%, lo cual complica la decisión sobre iniciar o no tratamiento antibiótico y obliga al médico tratante a valorar con objetividad su utilización ${ }^{9}$. Debido a la posible cura espontánea, recientemente la Academia Americana de Pediatría emitió sus guías para el manejo antimicrobiano en niños con otitis media, recomendando un tratamiento conservador, con analgésicos y sin antimicrobianos, en niños mayores de 24 meses de edad, sin factores de riesgo y con una otitis media leve ${ }^{11}$.

El éxito de la terapia antimicrobiana se basa, en gran medida, en el uso de medicamentos con actividad invitro frente a los agentes infecciosos involucrados en la OMA. La actividad antimicrobiana puede medirse de forma cuantitativa mediante la determinación de la concentración mínima inhibitoria (CMI) de los antibióticos frente a patógenos concretos. Sin embargo, desde la década de 1980 los conceptos farmacocinéticos y farmacodinámicos han complementado los conocimientos existentes y han mejorado la selección de tratamientos antibacterianos ${ }^{7,13}$.

El concepto de farmacocinética es el que evalúa los procesos que determinan la concentración de un antimicrobiano versus su tiempo en el compartimiento central y en los tejidos, entendiendo que la concentración antimicrobiana se alcanza a través de tres procesos: absorción, distribución y eliminación del fármaco. El conocer las características farmacocinéticas de un antibiótico permite determinar su vía de administración, dosificación y posibles ajustes según las vías de eliminación. La farmacodinamia, por otro lado, determina el efecto farmacológico y toxicológico del antimicrobiano de acuerdo a con su concentración en los tejidos y fluidos corporales, evaluando así el efecto terapéutico ${ }^{6}$.

El objetivo primordial del tratamiento antibacteriano de niños con otitis media es garantizar el éxito clínico con base en diversos aspectos fundamentales, como lo son: el espectro antibacteriano, las características farmacocinéticas y farmacodinámicas del medicamento, la erradicación bacteriana temprana del oído medio, la seguridad, el costo y la tolerabilidad. En la década de los 80 la selección de un tratamiento antimicrobiano se basaba en la necesidad de proveer una adecuada cobertura contra cepas de $H$. influenzae y $M$. catarrhalis productoras de $\beta$-lactamasas; sin embargo, en la actualidad la terapia empírica está dirigida a mantener la cobertura contra $H$. influenzae, pero a la vez a utilizar antibióticos con actividad contra $\mathrm{S}$. pneumoniae y, en particular, contra cepas de S. pneumoniae no susceptibles a penicilina ${ }^{10-12}$.

En el caso de los antibióticos, tres son los principales parámetros farmacodinámicos correlacionados con la eficacia terapéutica: el cociente inhibitorio (máxima concentración alcanzada en sangre / CMI), el área bajo la curva (ABC) durante 24 horas / CMI y el tiempo durante el cual las concentraciones del antibiótico a nivel sérico y a nivel del sitio de infección superan la $\mathrm{CMI}$ del patógeno $(\mathrm{T}>$ CMI).

Numerosos estudios clínicos y experimentales sugieren que alcanzar un óptimo cociente inhibitorio es esencial para garantizar la eficacia de los aminoglucósidos y fluoroquinolonas, mientras que una elevada proporción del ABC/CMI se correlaciona con la eficacia clínica de fluoroquinolonas y azitromicina, y el $\mathrm{T}>\mathrm{CMI}$ es el principal parámetro para antibióticos $\beta$ lactámicos, eritromicina y claritromicina (Cuadro 1) ${ }^{7,13}$.

\section{Desarrollo de resistencia a antibióticos}

La farmacodinamia es una herramienta necesaria para valorar el potencial de los antimicrobianos en la selección de resistencia antibiótica ${ }^{14,15}$. Recientemente se ha introducido un concepto denominado concentración preventiva de mutantes (CPM), que se fundamenta en la concentración de antibiótico capaz de prevenir que aparezca de una mutación de primer paso. De esta manera, los antibióticos con baja CPM previenen la selección y diseminación de bacterias resistentes.

Este concepto complementa otros parámetros de actividad antibacteriana, como la concentración inhibitoria mínima (CIM), y nos da una idea no solo de la actividad del antibiótico, sino de su capacidad para mantenerse activo

\begin{tabular}{|c|}
\hline $\begin{array}{c}\text { Cuadro 1. Modelo farmacodinámico de } \\
\text { distintos antimicrobianos }{ }^{6}\end{array}$ \\
\hline $\begin{array}{c}\text { Tiempo sobre la } \mathrm{CMI}(\mathrm{t}>\mathrm{CMI}) \\
\text { Penicilinas, cefalosporinas, monobactámicos, } \\
\text { carbapenems, macrólidos, clindamicina }\end{array}$ \\
Concentración máxima sobre la CMI (Cmáx/CMI) \\
Aminoglucósidos, quinolonas, metronidazol \\
Área bajo la curva sobre la CMI (ABC/CMI) \\
Aminoglucósidos, quinolonas azitromicina, \\
tetraciclina, vancomicina
\end{tabular}


frente a diversos gérmenes durante períodos prolongados. Cuando se administra un antibiótico, sus concentraciones plasmáticas aumentan hasta alcanzar la concentración pico, después disminuyen de forma progresiva y la velocidad de disminución va a condicionar su vida media plasmática. En el momento cuando las concentraciones plasmáticas son muy similares a las CMI, se alcanza el punto crítico en el que se pueden desarrollar resistencias ${ }^{14,16}$.

El concepto en cuestión es una medida que no aplica para todos los antimicrobianos, como por ejemplo para macrólidos y $\beta$ lactámicos, debido a que los mecanismos de resistencia valorados invitro para estos antibióticos, no se relacionan con los observados invivo ${ }^{14,16}$.

La verdadera importancia de la CPM reside en la ventana de selección de mutantes (VSM), concepto en el que la CPM participa como límite superior del intervalo de concentración dispuesto desde la CMI del microorganismo ${ }^{14}$.

El desarrollo de resistencia es una consecuencia inevitable de las estrategias de dosificación que sitúan las concentraciones del antimicrobiano dentro de la ventana de selección. Al evitar terapias que en forma continuada se sitúen dentro de la VSM, se podría minimizar el desarrollo de resistencia ${ }^{14}$. En el caso de los $\beta$ lactámicos el periodo durante el que concentraciones se encontrarían dentro de la ventana de selección sería mínimo, dada la proximidad de las medidas de CMI y CPM para las distintas especies de bacterias. Los $\beta$ lactámicos actúan de forma independiente de la concentración alcanzada, sin incrementos de actividad cuando esta aumenta en múltiplos de la CMI (Cuadro 2) ${ }^{14,17,18}$.

Los siguientes son los modelos farmacocinéticos y farmacodinámicos más estudiados y para los cuales existe evidencia científica que respalda su aplicación al seleccionar antibióticos para el tratamiento de la OMA en niños.

\section{Antimicrobianos dependientes del tiempo sobre la $\mathrm{CMI}(\mathrm{T}>\mathrm{CMI})$}

En este modelo, la eficacia de los antimicrobianos pertenecientes a este grupo se relaciona con el tiempo en que la concentración plasmática o tisular del fármaco es superior a la CMI. Diversos estudios han demostrado que para estos antimicrobianos, una vez que se logran concentraciones por encima de la CMI, se maximiza la capacidad de eliminación bacteriana y que esta aumenta discretamente hasta un límite sobre el cual no es posible obtener una mayor respuesta, a pesar de aumentar su concentración. La mayor parte de los datos se originan de estudios en modelos animales, sin embargo, en uno efectuado en niños con otitis media, utilizando antibióticos $\beta$ lactámicos, macrólidos (excepto azitromicina) y clotrimazol, se documentó entre un $80 \%$ y un $85 \%$ de eficacia clínica y microbiológica, cuando la concentración del antimicrobiano era superior a la CMI por un tiempo mayor al $40 \%$ o $50 \%$ del intervalo entre dos dosis, de esos medicamentos ${ }^{6,9}$.

Existe evidencia clínica de que la actividad antibacteriana de los miembros de este grupo depende del tiempo sobre la CMI; por ejemplo, se conoce que es posible alcanzar un efecto bacteriostático cuando el $\mathrm{T}>\mathrm{CMI}$ es de 30 a $40 \%$ el intervalo entre dos dosis, y un efecto bactericida si el T > CMI es de 60 a $70 \%$ del intervalo entre dos dosis (Figuras 1) ${ }^{6,9,13,19}$.

En un estudio clínico diseñado para comparar la eficacia del cefaclor versus la del cefuroxime axetil en la erradicación de cepas de $S$. pneumoniae y $H$. influenzae del oído medio, se logró demostrar como contra las cepas de $S$. pneumoniae sensibles a penicilina, en las cuales el T > CMI era $>50 \%$ se lograba una erradicación bacteriana $>90 \%$, mientras que contra cepas de $S$. pneumoniae no sensibles a

\begin{tabular}{|c|c|c|c|c|}
\hline Antibiótico & Dosis (mg/kg) & CMI $50(\mu \mathrm{g} / \mathrm{ml})$ & $\mathrm{t}>\mathrm{CMI} 50$ & $\begin{array}{l}\mathrm{CMl} 90(\%) \\
(\mathrm{mg} / \mathrm{ml})\end{array}$ \\
\hline Penicilina & $13.3 \times 3$ & 0.25 & $>70$ & 2 \\
\hline Amoxicilina & $13.3 \times 3$ & 0.25 & $>80$ & 4 \\
\hline Amoxicilina - clavulánico A & $13.3 \times 3$ & 0.25 & $>80$ & 4 \\
\hline Amoxicilina - clavulánico ${ }^{\mathrm{B}}$ & $26.6 \times 3$ & 0.25 & $>80$ & 4 \\
\hline Cefaclor & $13.3 \times 3$ & 4 & 0 & $\geq 64$ \\
\hline Cefuroxima & $15 \times 2$ & 1 & 37 & 8 \\
\hline Cefixima & $8 \times 2$ & 4 & 2 & 4 \\
\hline Eritromicina & $15 \times 3$ & $\leq 0.125$ & $>80$ & $\geq 64$ \\
\hline Claritromicina & $7.5 \times 2$ & $\leq 0.25$ & $>80$ & $\geq 64$ \\
\hline Azitromicina & $10 \times 1$ & $\leq 0.125$ & ND & $\geq 64$ \\
\hline
\end{tabular}




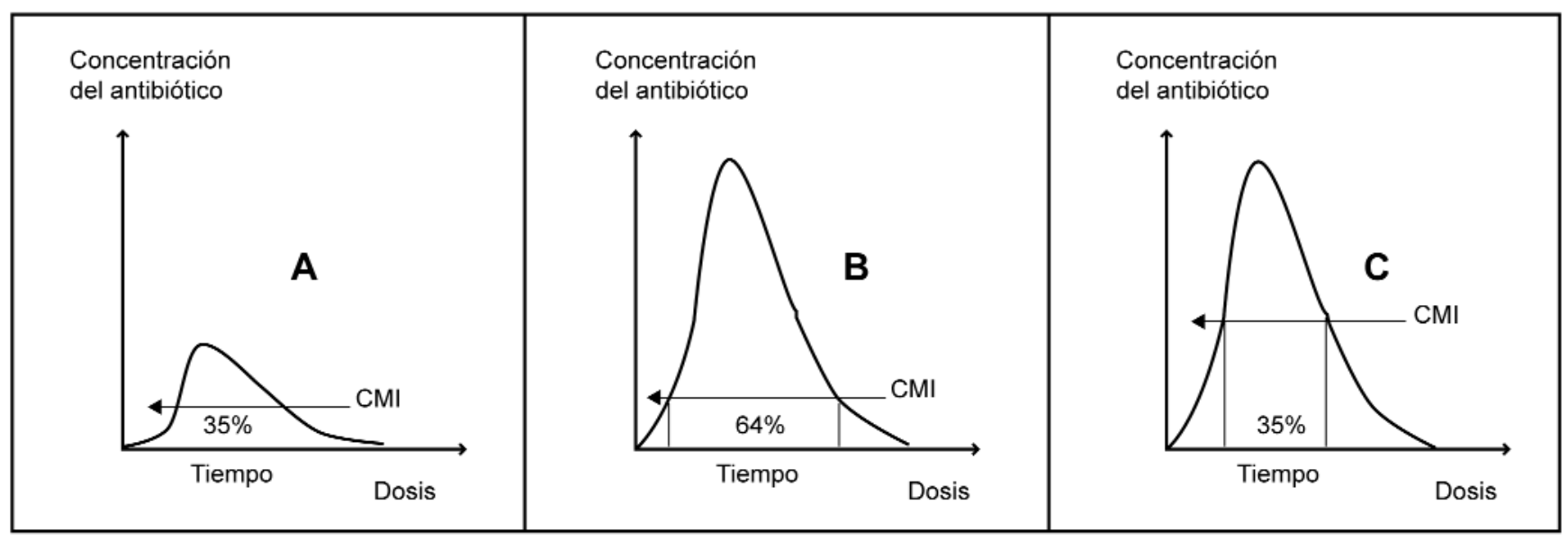

Figura 1. Comportamiento de antibióticos tiempo dependientes. A: El antibiótico no alcanza más del 35\% de tiempo sobre la concentración inhibitoria mínima del patógeno en el intervalo entre dos dosis, lo que minimiza la eliminación de patógeno y provoca una alta probabilidad de falla terapéutica en el paciente ${ }^{6,9,13}$. B: Cuando las concentraciones de antibiótico sobrepasan el $40-50 \%$ del tiempo sobre la concentración inhibitoria mínima del patógeno en el intervalo entre dos dosis, la probabilidad de erradicación del patógeno causante de la infección es mayor al $80-85 \%{ }^{6,9,13}$. C: Presenta un incremento en la CMI del patógeno, lo que provoca que este antibiótico (el mismo de B) no pueda en este caso erradicar al patógeno como si lo hizo en la figura anterior, pues en este caso la concentración de antibiótico no excede el $35 \%$ del tiempo sobre la CMl en el intervalo entre dos dosis ${ }^{6,9,13}$.

penicilina, en las que el $\mathrm{T}>\mathrm{CMI}$ era $<50 \%$, la erradicación bacteriana era inferior $(38 \%-79 \%)^{9,20,21}$.

\section{Antimicrobianos dependientes de la concentración C máx. /CMI}

La eficacia de los antibióticos con este modelo farmacocinético / farmacodinámico depende de la concentración plasmática del antimicrobiano sobre la CMI. Al incrementar la concentración del antibiótico, se aumenta el área bajo la curva, produciendo que estos antimicrobianos manifiesten un importante efecto postantibiótico ${ }^{6}$.

En el caso de los aminoglucósidos se ha demostrado que la concentración pico es el mejor predictor de la eficacia bacteriológica y clínica y también de la disminución de resistencia antimicrobiana, mientras que en el caso de las fluoroquinolonas, se ha concluido que la relación $\mathrm{C}$ máx. / CMI es un excelente parámetro para medir los resultados clínicos y de erradicación bacteriana ${ }^{6}$.

La correlación entre el coeficiente inhibitorio y la eficacia clínica se relaciona con las concentraciones del antibiótico. Se ha comprobado que cuando los cocientes inhibitorios son superiores a 10, la correlación con la eficacia clínica es excelente y cuando están entre 8-10, se puede prevenir la aparición de mutantes resistentes durante el tratamiento (Figura 2) ${ }^{13}$.

\section{Antimicrobianos dependientes de la concentración $\mathrm{ABC} / \mathrm{CMI}$}

Este modelo mide la exposición total de la bacteria frente a un antimicrobiano. Corresponde a una combinación de la concentración pico del antibiótico con el tiempo de exposición a niveles sobre la CMI. Esta relación es depen-

\section{Concentración \\ del antibiótico}

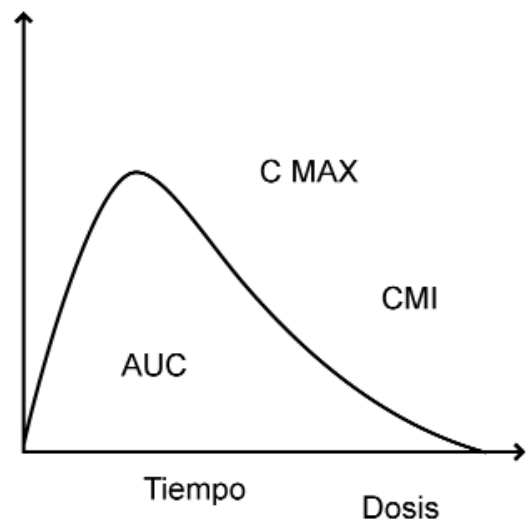

Figura 2. Relación farmacológica para antibióticos de la familia de las quinolonas y azalidos. Al incrementar la concentración del antibiótico, se aumenta el área bajo la curva, lo que produce que estos antimicrobianos manifiesten un importante efecto postantibiótico ${ }^{6}$. Se ha comprobado que cuando los cocientes inhibitorios son superiores a 10, la correlación con la eficacia clínica es excelente y cuando están entre $8-10$, se puede prevenir la aparición de mutantes resistentes durante el tratamiento ${ }^{13}$.

diente de la Cmáx. del antibiótico y es importante en fármacos con una vida media larga (ej. azitromicina) ${ }^{6}$.

Numerosos modelos experimentales han demostrado una buena correlación de eficacia cuando el cociente $\mathrm{ABC} /$ CMI es $>25$ para infecciones leves a moderadas y $>125$ para infecciones severas o en niños inmunocomprometidos. En el caso de las fluoroquinolonas y de la azitromicina, en infecciones producidas por el $S$. pneumoniae se ha observado una correlación muy estrecha con éxito terapéutico, 
cuando este cociente es $>30$, y al mismo tiempo se ha visto que valores $<30$ se asocian con una reducción del efecto bactericida y, en algunos casos, con recrecimiento bacteriano ${ }^{13,22-25}$. En el caso de la azitromicina, que presenta una $\mathrm{ABC}$ de $3 \mathrm{mg} . \mathrm{h} / \mathrm{L}$, su límite farmacodinámico en niños inmunocompetentes es de $0.12 \mathrm{mg} / \mathrm{L}$, por lo que para infecciones por S. pneumoniae susceptibles a macrólidos, los resultados clínicos serían adecuados, ya que la CMI para estos gérmenes es de $0.12 \mathrm{mg} / \mathrm{L}$, no así para $H$. influenzae o para S. pneumoniae resistentes a macrólidos, en donde la CMI es de $1-2 \mathrm{mg} / \mathrm{L} \mathrm{y}>8 \mathrm{mg} / \mathrm{L}$, respectivamente (Figura 3) ${ }^{25-27}$.

\section{Efecto postantibióticos}

Este término se refiere al tiempo requerido para que determinados microorganismos se recuperen y vuelvan a la fase logarítmica de crecimiento, después de haber estado expuestos a determinado antibiótico. El fenómeno es microorganismo y antimicrobiano dependiente. Aquellos fármacos que actúan por concentración máxima tienen un mayor efecto postantibiótico (EPA), como es el caso de los aminoglucósidos y ciprofloxacina, con un EPA contra bacilos Gram negativos que supera las 8 horas, lo cual explica por qué los aminoglicósidos pueden ser utilizados con una dosificación total dividida en intervalos de 24 horas para el manejo de ciertas infecciones urinarias ${ }^{6}$.

Los antibióticos $\beta$-lactámicos no tienen EPA contra bacilos Gram negativos y este es tan solo de 2 horas contra bacterias Gram positivas. Teóricamente, es posible modificar la dosificación de los antimicrobianos para aprovechar el EPA, pudiendo en aquellos fármacos con EPA prolongado, aumentar el intervalo entre dosis, mientras que para antimicrobianos con EPA corta se deben valorar intervalos cortos o infusiones continuas ${ }^{6}$.

\section{Correlación entre erradicación bacteriológica y éxito terapéutico en niños con OMA}

Es importante reconocer que existe una relación ampliamente documentada entre éxito bacteriológico (erradicación durante tratamiento) y éxito clínico al final de tratamiento, en donde un antibiótico con una pobre erradicación bacteriológica durante tratamiento, suele asociarse con un alto nivel de fallas clínicas y de recaídas. Este concepto ha sido demostrado en varios estudios clínicos que emplean el diseño de doble timpanocentesis, en los que se ha documentado que los niños con persistencia bacteriológica durante tratamiento tienen hasta 3 veces más la posibilidad de una falla clínica al final de tratamiento, que el grupo de quienes presentaron una erradicación bacteriológica durante tratamiento. De la misma forma, estudios recientes han demostrado una mayor posibilidad de recaída clínica en los niños con persistencia bacteriana ${ }^{9,28-31}$.

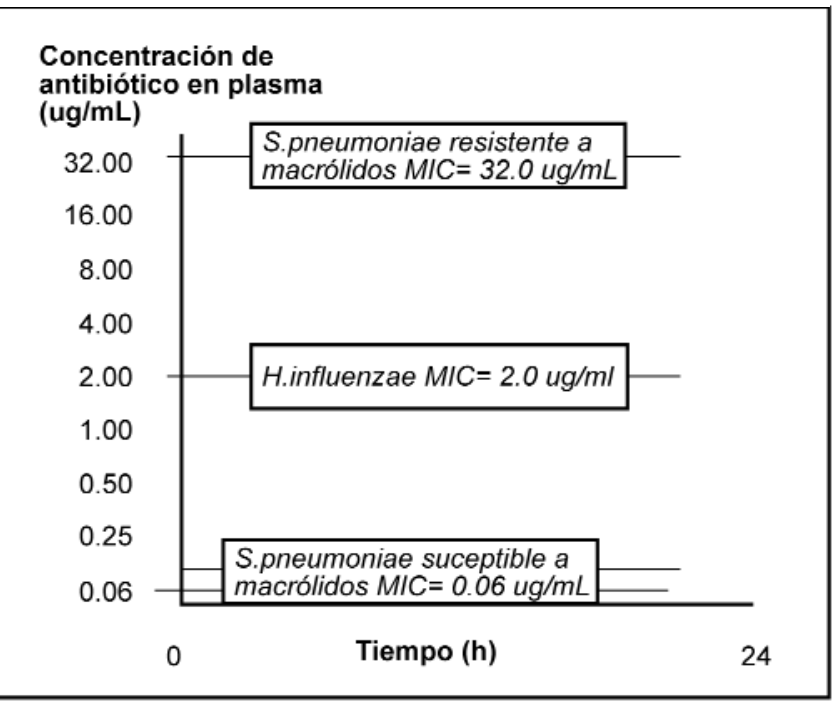

Figura 3. Relación farmacológica para la azitromicina. Niños con infecciones causadas por $S$. pneumoniae susceptibles a macrólidos responden bien a la azitromicina, pues este patógeno tiene una MIC de $0.06 \mu \mathrm{g} / \mathrm{mL}$ y la azitromicina tiene una buena cobertura sobre él mismo, no así para patógenos como $\mathrm{H}$. influenzae o $S$. pneumoniae resistente a macrólidos, con unas MIC que sobrepasan la cobertura del antibiótico ${ }^{25-27}$.

La OMA en niños es un problema de gran importancia médica y social, por lo que el diagnóstico clínico, el conocimiento microbiológico de las bacterias que causan este proceso infeccioso y su comportamiento antimicrobiano son elementos fundamentales.

La valoración de nuevos antibióticos para el tratamiento de niños con otitis media debe aplicar y conjugar conceptos de farmacocinética y farmacodinamia con estudios clínicos que involucren una segunda timpanocentesis durante tratamiento (días 4 a 6), para valorar adecuadamente la eficacia bacteriológica y la respuesta clínica de nuevos antibióticos.

\section{Abstract}

Because acute respiratory infections and particularly acute otitis media (AOM), are the most common cause of antimicrobial prescription in pediatric patients, it is important to optimize antimicrobial therapies. It is essential that before prescribing an antimicrobial agent, the AOM diagnosis is well established and the local microbiological pattern is known. The present review incorporates novel concepts for the selection of the antimicrobial therapy in children with AOM taking into account pharmacokinetic and pharmacodynamic principles applied to microbiologycal concepts. These new concepts have revolutionized the treatment of diverse infectious diseases in pediatric patients and particularly in the treatment of children with otitis media. 


\section{Referencias}

Álvarez L. Otitis media aguda: nuevo enfoque terapéutico. Rev Cubana Pediatr 2004; 76:0-0

2. López FJ. Epidemiología de las infecciones respiratorias agudas en niños: panorama regional. En: Benguigui Y. Infecciones respiratorias en niños. Washington, DC, 1997: 3-20.

3. Faden H, DuffyL, Boeve M. Otitis media: back to basics. Pediatric Inf Dis J 1998; 17: 1105-12.

4. Arguedas A, Dagan R, Soley C, Loaiza C, Knudsen K, Porat N, et al. Microbiology of otitis media in Costa Rican children, 1999 through 2001. Pediatr Infect Dis J 2003; 22: 1063-8

5. Sierra H, Schultz M, Loaiza C, Arguedas A. Otitis media: conceptos actuales. Acta med. Costarric 2004; 46: 125-131.

6. Labarca J. Nuevos conceptos en farmacodinamia: ¿Debemos repensar como administrar antimicrobianos?. Rev. Chil. Infectol. 2002; 19: 33-37.

7. Barberán, J. Farmacodinamia como predictor de eficacia: la visión del clínico. Rev Esp Quimioterap. 2005; 18: 63-64

8. Guevara S, Soley C, Arguedas A. Seasonal patterns of otitis media pathogens distribution in Costa Rica Children during period 19992004.

9. Dagan R, Leivovitz E. Bacterial eradication in the treatment of otitis media. The Lancet Infection Diseases. 2002;2: 593-604

10. Soley C, Arguedas A. Sigle-dose azithromycin for the treatment of children with acute otitis media. Expert Rev. Anti Infect. Ther. 2005; 3: 707-717

11. American Academy of Pediatrics, American Academy of Family Physicians, subcommittee on management of acute otitis media. Diagnosis and management of acute otitis media. Pediatrics. 2004; 113: $1451-1465$

12. García E. Farmacocinéticas/Farmacodinamia y el desarrollo de nuevas formulaciones en el campo de los antimicrobianos. Rev Esp Quimioterap. 2005; 18: 65-69

13. Soriano F. Farmacodinamia: factor predictivo de eficacia. Anales Españoles de Pediatría. 2002; 56: 25-30

14. Sevillano D, Jiménez M.J, Aguilar L, Prieto J. ¿Es la farmacocinética una herramienta útil para la prevención de las resistencias? Rev Esp Quimioterap. 2005 18: 77-79

15. Zhao X, Drlica K, Restricting the selection of antibiotic-resistant mutant bacteria: Measurement and potential use of the mutant selection window. J Infect Dis. 2002; 185: 561-565.

16. Smith H.J, Nichol K.A, Hoban D.J, Zhanel G.G. Stretching the mutant prevention concentration (MPC) beyond its limits. J Antimicrob Chemother 2003; 51:1323-1325

17. Zhao X. Clarification of MPC and the mutant selection window concept. J. Antimicrob Chemother 2003; 52: 731

18. Hovde L, Rotchasfer E, Ibrahim H, Gunderson B, Hermsen D, Rotchafer C. Mutation prevention concentration of ceftriaxona, meropenen, imipenen, and ertapenen against three strains of Streptococcus pneumoniae . Diagn Microbiol Infect Dis 2003; 45: 265-567.
19. Howie VM. Otitis media. Pediatr Rev 1993; 14: 320-23

20. Dagan R, Abramson O, Leibovitz E, Greenberg D, Lang R, Goshen $\mathrm{S}$, et al. Bacteriologic response to oral cephalosporins: Are established susceptibility breakpoints appropriate in the case of acute otitis media? J Infect Dis 1997; 176:1253-59

21. Dagan R, Leibovitz E, Fliss DM, Leiberman A, Jacobs MR, Craig W, et al. Bacteriologic efficacy of oral azithromycin and oral cefaclor in treatment of acute otitis media in infants and young children. Antimicrob Agents Chemother 2000; 44: 43-50.

22. Fung-Tomc J, Kolek B, Bonner DP. Ciprofloxacin - induced, low level resistance to structurally unrelated antibiotic in Pseudomonas aeruginosa and methicillin- resistant Staphylococcus aureus. Antimicrob Agents Chemother 1993; 37: 1289-1296.

23. Coyle EA, Rybak MA. Evaluation of the activity of the newer fluoroquinolonas against ciprofloxacin - resistant Streptococcus pneumoniae. (Abstract 18, p 5). In: 39th Interscience Conference on Antimicrobial Agents and Chemotherapy, San Francisco, CA, 1999

24. Lacy MK, Lu W, Xu X, Tessier PR, Nicolau DP, Quintilliana R el al. Pharmacodynamic comparisons of levofloxacin, ciprofloxacin, and ampicillin against Streptococcus pneumoniae in an in-vitro model of infection. Antimicrob Agents Chemother 1999; 43: 672-677

25. Jacobs R. Optimisation of antimicrobial therapy using pharmacokinetic and pharmacodynamic parameters. Clin Microbiol Infect 2001; 7: 589-596.

26. Dagan R, Leibovitz E, Leiberman A, Yagupsky D. Clinical significance of antibiotic resistance in acute otitis media and implication of antibiotic treatment on carriage and spread of resistance organisms. Pediatr Infect Dis J 2000; 19:S57-65.

27. Dagan R, Johnson CE, McLinn S et al. Bacteriologic and clinical efficacy of amoxicillin / clavulanate vs. azithromycin in acute otitis media. Pediatr Infect Dis J 2000; 19: 95 - 104.

28. Dagan R, Leibovitz E, Greenbert D, Yagupsky P, Fliss D, Leiberman A. Early eradication of pathogens from middle ear fluid during antibiotic treatment of acute otitis media is associated with improved clinical outcome. Pediatr Infect Dis J 1998; 17: 776-782.

29. Asher E, Greenberg D, Dagan R, Porat N, Leibovitz E. Failure to eradicate otitis media pathogens after 3-5 days of treatment is associated with higher relapse rate in children with initial improvement / cure. (Abstract G-2096, p. 271). In: Program and Abstracts of the 44th Interscience Conference on Antimicrobial Agents and Chemotherapy, October 30-November 2, 2004, Washington, DC.

30. Dagan R, McCracken GH. Flaws in design and conduct of clinical trial in acute otitis media. Pediatr Infect Dis J 2002:21:894-902.

31. Dagan R, Leibovitz E, Arguedas A, Hoberman A, Pichichero M, Buscan T, Paris M, Bucofzer S. The Clinical and bacteriological efficacy of a once daily (OD) increased dose (ID) $(25 \mathrm{mg} / \mathrm{kg}$ ) of cefdinir $(\mathrm{CEF})$ in children with acute otitis media (AOM) at high risk of persistent/recurrent otitis media (HR-PROM). (Abstract 1121, p 259). In: 44th Interscience Conference on Antimicrobial Agents and Chemotherapy, Washington, D C, October 2004. 